\section{DGS begrüßt das Anti-Korruptionsgesetz}

Am 4. Juni 2016 ist das „Gesetz zur Bekämpfung von Korruption im Gesundheitswesen" in Kraft getreten. Die Deutsche Gesellschaft für Schmerzmedizin e.V. (DGS) begrüßt das Gesetz sehr. Denn ab sofort können Patienten darauf vertrauen, dass Ärzte ausschließlich das Patientenwohl im Blick haben - ohne persönliche, wirtschaftliche oder andere Interessen. Einflussnahme und Bestechung, so Dr. Gerhard H. H. Müller-Schwefe, Präsident der DGS, sind nicht nur das Überlassen von Praxisartikeln oder ein umfangreiches Rahmenprogramm bei Kongressen.

Der DGS war das auf den eigenen Vorteil bedachte Gebaren schon immer ein Dorn im Auge: „Es ist absolut richtig, dass Bestechung und Bestechlichkeit geahndet werden - generell und besonders in der Medizin. Ein Arzt, der sich persönlich bereichern will, hat seine ethische Verpflichtung vergessen", sagte Müller-Schwefe. Der Eid des Hippokrates ist für die DGS Richtschnur allen Handelns: „Wir als VersorgerGesellschaft haben das Wohl des Patienten im Blick und nicht die Gewinnmaximierung. Wir verschreiben das Medikament, das am besten hilft, und empfehlen Behandlungen, die erfolgversprechend sind", erklärte MüllerSchwefe.

Neben fachlichen Kompetenzen sind gerade bei der Arbeit mit Schmerzpatienten vor allem menschliche Kompetenzen das A und O. Das Leben chronischer Schmerzpatienten besteht häufig aus einer Aneinanderreihung von Katastrophen: gestörte soziale Bindungen, Arbeitsplatzverlust, Probleme in der Partnerschaft und vieles mehr. Oft haben diese Patienten kein Vertrauen mehr, weil sie die Erfahrung gemacht haben, dass ihnen ihre Wahrnehmung der chronischen Schmerzkrankheit nicht geglaubt wird. „Um dieses Vertrauen zurückzugewinnen, ist ein hohes Maß an Einfühlungsvermögen und kommunikativer Kompetenz nötig", so Müller-Schwefe. Das Vertrauensverhältnis zwischen Arzt und Patient sieht der DGS-Präsident durch das Anti-Korruptionsgesetz nun noch stärker geschützt, "weil sichergestellt wird, dass eine bestimmte Behandlung allein aus medizinischen Gründen erfolgt".

Quelle: Pressemitteilung der DGS
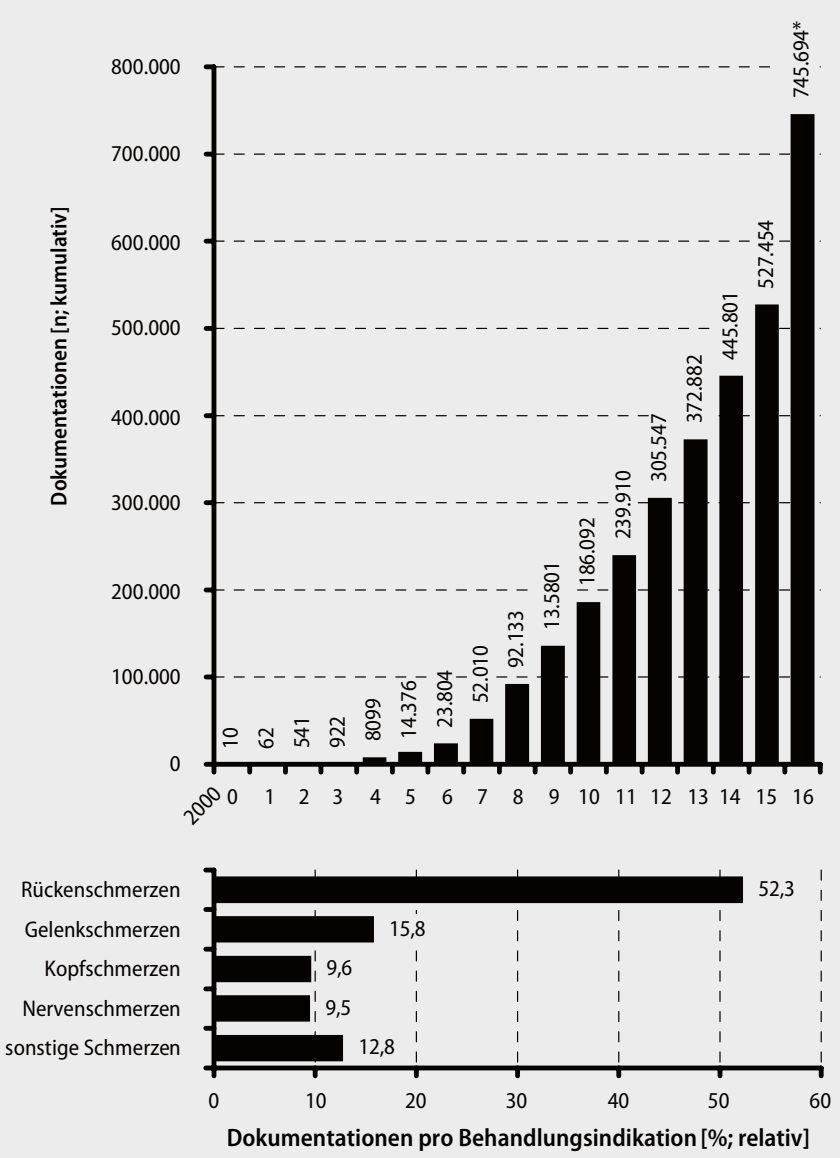

Abb. 2: Entwicklung der Behandlungsdokumentationen im DGS-PraxisRegister (oben) sowie Umfang der Dokumentationen pro Behandlungsindikation (unten).

*Anmerkung: Hochrechnung 2016 auf Grundlage der aktuellen Entwicklung bis Ende April 2016; ohne Daten der Patientenplattform mein-Schmerz.de (aktueller Stand zum 30.04.2016: $n=107.852$ )

lichkeit einzelner Behandlungsverfahren unter Alltagsbedingungen hinterfragt werden. Auf diesem Weg wird nicht nur ein seit langem beklagtes Informationsdefizit behoben, sondern es entsteht gleichzeitig auch ein Informationskomplementär zu den Daten aus kontrollierten klinischen Prüfungen und Studien.

\section{Nicht nur hilfreich für die Regelversorgung}

Aufgrund seiner alltagskonformen Konzeption ermöglicht das DGS-PraxisRegister Schmerz und die ihm zugrunde liegende online Dokumentationsplattform iDocLive ${ }^{\circledR}$ nicht nur die Begleitung alltäglicher Versorgungsfragestellungen, sondern auch die gezielte Evaluation spezifischer Behandlungssituationen wie etwa die wissenschaftliche Begleitung des Einsatzes neuer Therapien unter Alltagsbedingungen. So ließen sich zum Beispiel nicht nur der aktuell vielfach diskutierte Einsatz von Cannabis für die Behandlung chronischer Schmerzen evaluieren, sondern auch zeitnah nach Zulassung repräsentative Daten zum Zusatznutzen neuer Therapien unter Alltagsbedingungen erheben.

PD Dr. med. Michael A. Überall, Nürnberg 